\title{
Comparison of holographic lens and filter systems for lateral spectrum splitting
}

\author{
Shelby Vorndran ${ }^{\mathrm{a}}$, Benjamin Chrysler $^{\mathrm{b}}$ Raymond K. Kostuk ${ }^{\mathrm{a}, \mathrm{b}^{*}}$ \\ ${ }^{a}$ College of Optical Sciences, University of Arizona. Tucson, AZ 85721 \\ ${ }^{b}$ Department of Electrical and Computer Engineering, University of Arizona, Tucson, AZ 85721
}

\begin{abstract}
Spectrum splitting is an approach to increasing the conversion efficiency of a photovoltaic (PV) system. Several methods can be used to perform this function which requires efficient spatial separation of different spectral bands of the incident solar radiation. In this paper several of holographic methods for implementing spectrum splitting are reviewed along with the benefits and disadvantages associated with each approach. The review indicates that a volume holographic lens has many advantages for spectrum splitting in terms of both power conversion efficiency and energy yield. A specific design for a volume holographic spectrum splitting lens is discussed for use with high bandgap InGaP and low bandgap silicon PV cells. The holographic lenses are modeled using rigorous coupled wave analysis, and the optical efficiency is evaluated using non-sequential raytracing. A proof-of-concept off-axis holographic lens is also recorded in dichromated gelatin film and the spectral diffraction efficiency of the hologram is measured with multiple laser sources across the diffracted spectral band. The experimental volume holographic lens (VHL) characteristics are compared to an ideal spectrum splitting filter in terms of power conversion efficiency and energy yield in environments with high direct normal incidence (DNI) illumination and high levels of diffuse illumination. The results show that the experimental VHL can achieve $62.5 \%$ of the ideal filter power conversion efficiency, $64.8 \%$ of the ideal filter DNI environment energy yield, and $57.7 \%$ of the ideal diffuse environment energy yield performance.
\end{abstract}

Keywords: Solar energy, holographic optical elements, spectrum splitting

\section{INTRODUCTION}

Detailed balance considerations show that the maximum efficiency of a single bandgap PV cell is $\sim 33 \%{ }^{1}$. This limit can be overcome by using multiple bandgap PV cells that span the broad incident solar spectrum. Multi-junction tandem PV cells have been used with high concentration solar collectors with some success. However, series connected cells are current limited by lowest output bandgap in the stack. In addition, precise lattice matching is required at each interface between different bandgaps in the multi-junction cell. An alternative approach is to spatially separate different spectral bands of the incident solar illumination and direct them onto PV cells with high responsivity to a specific spectral band ${ }^{2,3}$. This approach is not current limited and can use different material systems providing much more flexibility in design and the potential for higher energy yield.

The main challenge for spectrum splitting systems is to provide sufficient improvement in efficiency and energy yield performance to offset the higher complexity of the optical system. The main result is to reduce the cost per performance ratio in terms of $\$ / \mathrm{W}$ or $\$ / \mathrm{kW}$-hr. In this paper a review of different approaches to spectrum splitting and illustrate how an approach using a volume holographic lens can satisfy the requirements for higher efficiency with low complexity of the optical system.

\section{PERFORMANCE METRICS AND PV CELL SELECTION}

Several parameters are important in the evaluation of spectrum splitting system performance. The basic goal is to effectively combine PV cells to produce a significant increase in the overall system conversion efficiency. The conversion of a single cell is given by:

*Further author information: R. K. K.: e-mail: kostuk@email.arizona.edu: Phone: (520) 621-6172

Next Generation Technologies for Solar Energy Conversion VII, edited by Oleg V. Sulima,

Gavin Conibeer, Proc. of SPIE Vol. 9937, 99370K - (C) 2016 SPIE

CCC code: $0277-786 \mathrm{X} / 16 / \$ 18 \cdot$ doi: $10.1117 / 12.2236105$

Proc. of SPIE Vol. 9937 99370K-1 


$$
\eta_{k}=\frac{P_{O U T-P V}^{k}}{P_{I N-\text { optical }}},
$$

where $P_{O U T-P V}^{k}$ is the electrical output power of the $\mathrm{k}^{\text {th }}$ cell in the system and the total incident optical power is

$$
P_{I N-\text { optical }}=\int E_{I N}(\lambda) \cdot d \lambda,
$$

with $E_{i n}(\lambda)$ the solar spectral irradiance. The total electrical output power is related to the spectral conversion efficiency $(\operatorname{SCE}(\lambda))$ of the cell':

$$
\operatorname{SCE}^{k}(\lambda)=S R^{k}(\lambda) \cdot V_{o c}^{k} \cdot F F^{k}
$$

where $S R^{k}(\lambda), V_{o c}^{k}$, and $F F^{k}$ are respectively the spectral responsivity, open circuit voltage, and fill factor for the $k^{\text {th }}$ cell. The SCE represents the combined optical absorption, bandgap, and circuit characteristics of the PV cell and is a good indicator for comparing cells in a spectrum splitting system. Figure 1 shows the AM 1.5 spectral irradiance along with the ideal SCE for silicon, GaAs, and InGaP PV cells. If two cells were used in a PV system, the best combination for the three cells shown would be silicon and InGaP since they have the larger combined increase in SCE. Notice that even for ideal PV cell there will always be some degree of overlap of SCE at wavelengths less than the bandgap due to the linear spectral responsivity characteristic.

The short circuit current produced by a PV cell is given as:

$$
J_{s c}^{k}=\int E_{A M 1.5}(\lambda) \cdot S R^{k}(\lambda) \cdot d \lambda=\frac{q}{h c} \int \lambda \cdot E_{A M 1.5}(\lambda) \cdot E Q E^{k}(\lambda) \cdot d \lambda,
$$

where $E_{A M 1.5}(\lambda)$ is the incident AM 1.5 solar spectral irradiance, $S R^{k}$ is the spectral responsivity, and EQE is the external quantum efficiency of the PV cell. The power produced by the cell is:

$$
P_{\text {OUT }-P V}^{k}=\int E_{A M 1.5}(\lambda) S R^{k}(\lambda) V_{o c}^{k} F F^{k} d \lambda=\int E_{A M 1.5}(\lambda) S C E^{k}(\lambda) d \lambda .
$$

The optics that spatially separates the different spectral bands can be expressed as a transmittance function $T(\lambda)$ and directly incorporated into the cell conversion efficiency:

$$
\eta_{k}^{*}=\frac{1}{P_{A M 1.5}} \int T_{k}(\lambda) \cdot E_{A M 1.5}(\lambda) \cdot S C E_{k}(\lambda) \cdot d \lambda .
$$

If $M$ different PV cells are used in the spectrum splitting system, the cumulative efficiency can be written as:

$$
\eta_{\text {SSS }}=\sum_{k=1}^{M} \eta_{k}^{*}
$$

The total spectrum splitting system efficiency can then be compared to other PV systems to determine how much of an efficiency gain is possible. Another metric for spectrum splitting systems is the improvement it provides over a similar size system with best performing single bandgap cell ${ }^{4}$. This parameter is the improvement over best bandgap or (IoBB) where:

$$
I o B B=\frac{\eta_{S S S}}{M A X\left[\eta_{1}, \eta_{2} \cdots \eta_{2}\right]}-1
$$

\section{APPROACHES TO SPECTRUM SPLITTING}

Several different approaches to spectrum splitting are possible. Figure 2 shows basic forms of reflection and transmission type spectrum splitting systems. For a reflection system spectral bandwidth filters divert a component of the incident bandwidth that matches the response characteristic of a particular PV cell. As the incident solar spectrum propagates through the system additional spectral components are extracted. For this particular system $N-1$ spectral filters are required for a spectrum splitting system with $N$ different PV cell bandgaps. Many reflection type 
spectrum splitting systems require additional area to accommodate the PV cells mounted off-axis. This reduces the packing density and the effective conversion efficiency.

A transmission type spectrum splitting system is shown in Fig. 2B. In this case the longer wavelengths in the incident spectrum over the high bandgap cell is diffracted to the narrow bandgap cell and in the area of the narrow bandgap cell higher energy photons are diffracted to the high bandgap cell. However, one of the problems with this type of transmission spectrum splitting system is the spatial-spectral dispersion. As shown in Fig. 3 light is dispersed at each point along the aperture of the filter. Therefore, different spectral components overlap at the receiver plane except at the edge of the receiver. One way to eliminate spectral overlap is to incorporate focusing into the system. When broadband light is normally incident on a grating or holographic dispersive filter each wavelength is diffracted at a different angle according to the grating equation:

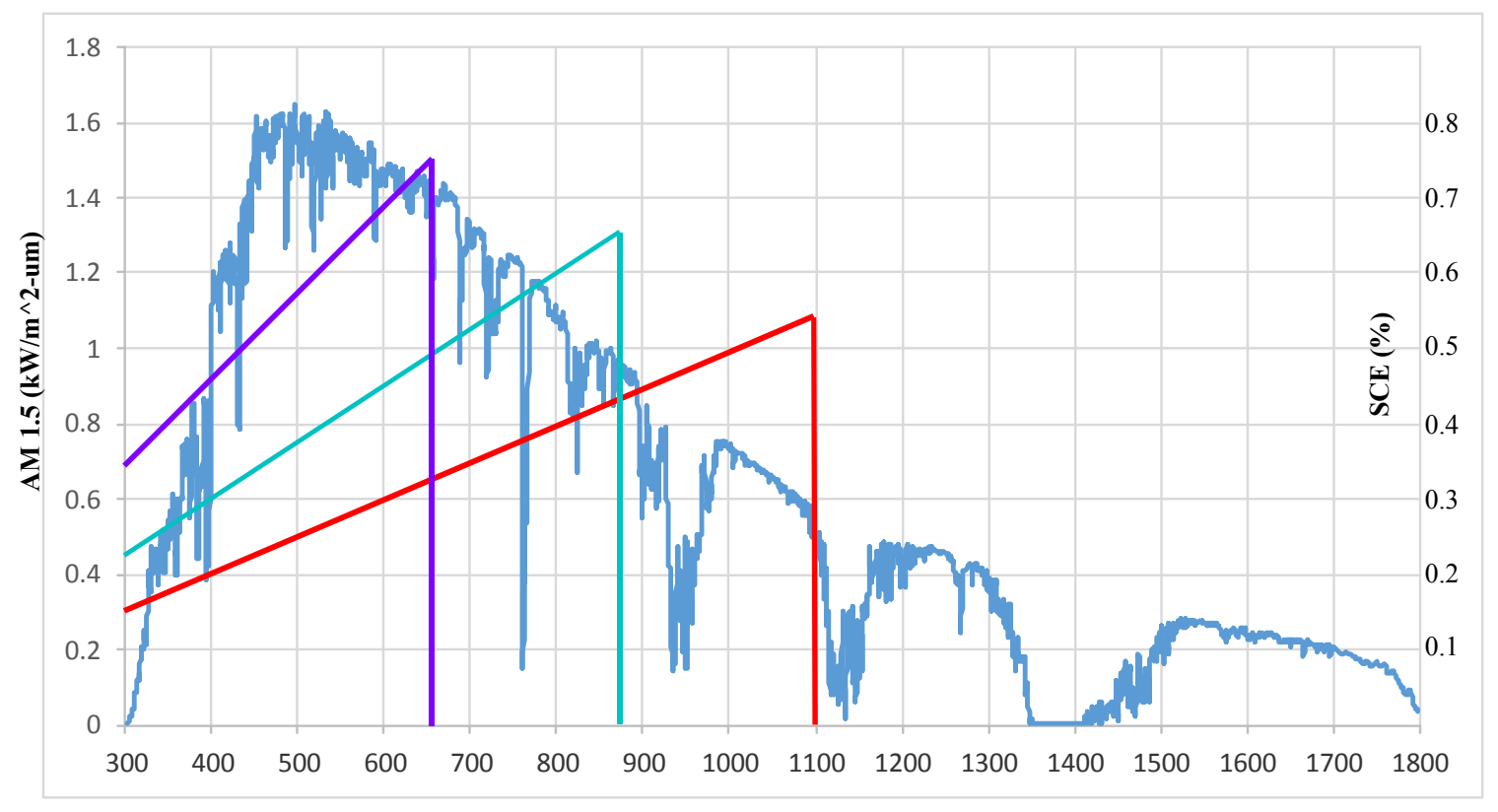

Wavelength (nm)

Fig. 1. AM 1.5 solar spectral irradiance and ideal SCE for silicon (red), GaAs (green-blue), and InGaP (purple) type PV cells.

$$
\sin \theta_{i}=\frac{\lambda_{i}}{\Lambda}
$$

where $\Lambda$ is the grating period, $\lambda_{\mathrm{i}}$ is an incident wavelength, and $\theta_{\mathrm{i}}$ is the angle that the incident wavelength is diffracted with respect to the normal to the hologram. Since light with the same wavelength is diffracted at the same angle a lens will focus each wavelength to a different focal position at the back focus of the lens 5 .

Focusing can readily be incorporated into a hologram forming a holographic lens ${ }^{6}$. This can provide a very compact dispersive filter for spectrum splitting applications. One configuration is to design the holographic lens with the focus converging light at the wavelength at the transition between the high and low bandgap for the PV cells used in the system ${ }^{7}$. The volume holographic lens (VHL) design of this type is illustrated in Fig. 4. As shown from the grating equation (Eq. 1) wavelengths shorter than the transition wavelength are diffracted at smaller angles relative to the VHL normal whereas longer wavelengths are diffracted at larger angles. In addition, since volume gratings are Bragg selective ${ }^{8}$ light that is not within the coupling range of the grating will pass through the hologram providing a mechanism for capturing diffuse illumination and increasing the energy yield of the system. 

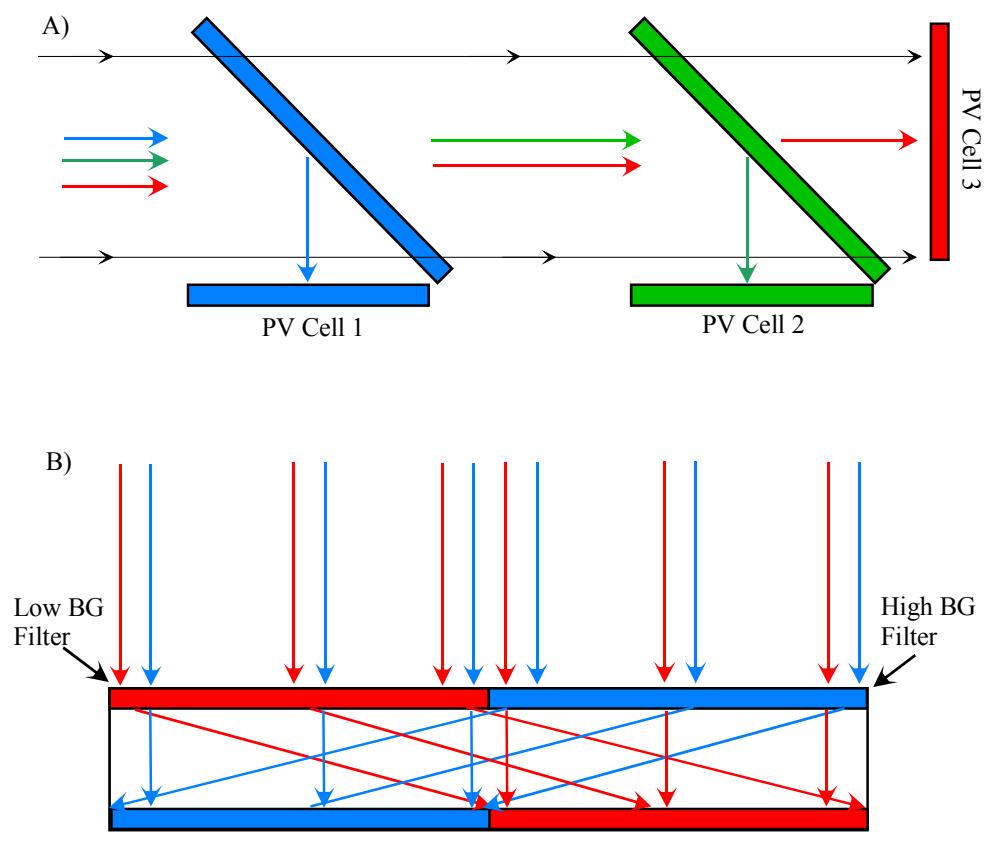

PV Cell (High BG)

PV Cell (Narrow BG)

Fig. 2. Figure A shows a reflection type spectrum splitting system and B) shows a transmission type spectrum splitting system.

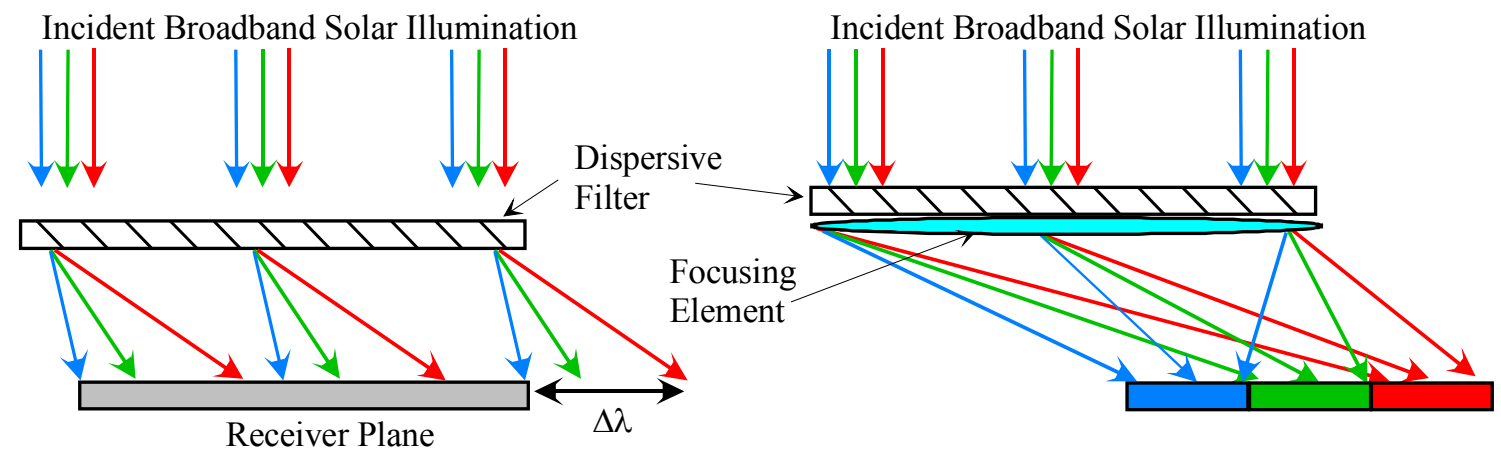

Fig. 3. The left illustration shows the effect of dispersion from a transmission type dispersive filter resulting in overlapping wavelengths along the receiver plane. Adding a lens (right figure) in combination with the filter separates wavelengths along the receiver plane.

\section{VOLUME HOLOGRAPHIC LENS DESIGN}

In order to be effective as a spectrum splitting filter a volume holographic lens (VHL) must satisfy several properties. First the diffracted rays much reach the designated PV cell surface and each ray must have high diffraction efficiency across the full aperture of the hologram. This allows high optical efficiency which is necessary for high PV conversion efficiency. In addition, high diffraction efficiency must be realized for the range of optical wavelengths corresponding to the response spectrum of a particular PV cell. The spectral diffraction efficiency should also have a sharp transition in transmittance between the low and high bandgap PV cells. Finally, the VHL should also allow diffuse illumination to pass through the element and allow collection to increase the effective energy yield.

Focusing in a holographic lens is achieved by varying the grating period across the aperture. For the spectrum splitting designs considered in this paper focusing will only be performed in one direction similar to that of conventional cylindrical lens. In order to achieve high diffraction efficiency, the grating parameters of the VHL must satisfy the following condition ${ }^{8}$ : 


$$
Q=\frac{2 \pi \lambda_{\min } d}{n \Lambda_{\max }^{2}} \geq 10
$$

where $\lambda_{\min }$ is the minimum wavelength in the diffracted spectral band, $d$ is the hologram thickness, $n$ is the average refractive index of the hologram, and $\Lambda_{\max }$ is the maximum grating period across the aperture.

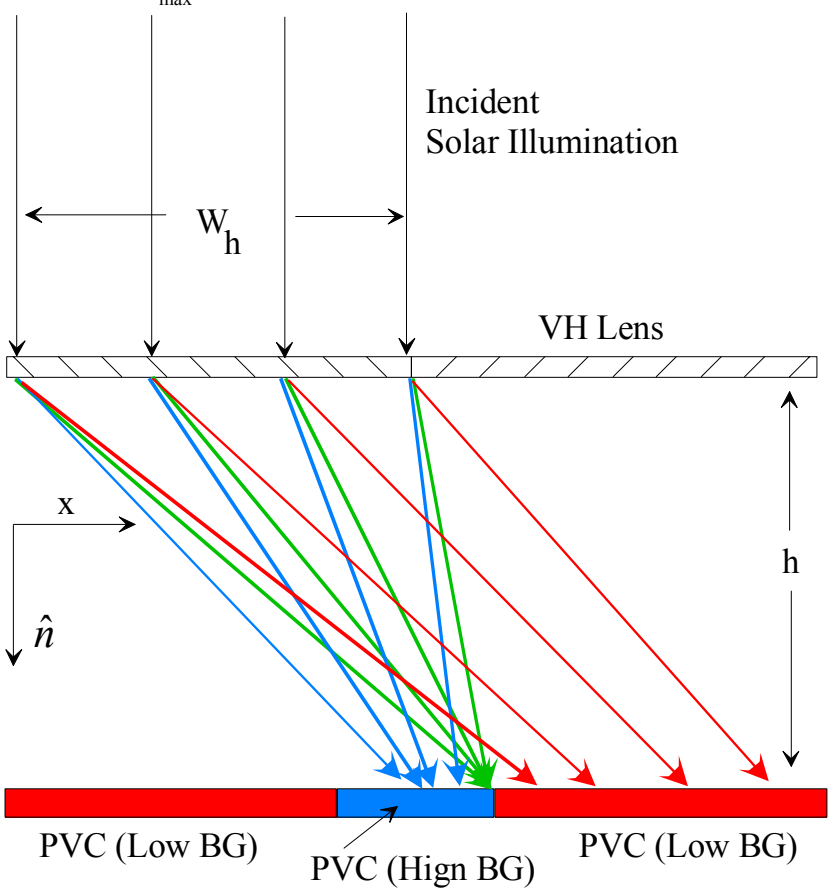

Fig.4. A volume holographic lens that focuses light at the transition wavelength to the point separating the high and low bandgap PV cells.

The direction of diffracted rays is determined by the surface grating at the interface between the volume grating and the surrounding material. If $\mathrm{x}$ is considered the direction along the grating surface in which the grating period is varied, then the diffracted ray directions can be determined using the grating equation:

$$
\sin \theta_{i n c}-\sin \theta_{i}(x)=\frac{\lambda_{i}(x)}{\Lambda_{x}(x)}
$$

where $\theta_{i n c}$ is the angle of incidence, $\theta_{i}(x)$ is the diffracted angle at a particular $\mathrm{x}$ location along the aperture, $\lambda_{i}(x)$ is the diffracted wavelength at the x-location, and $\Lambda_{x}(x)$ is the $\mathrm{x}$ component of the grating period at the position $x$.

The basic VHL design approach is to construct the hologram with the point of focus located at the point of separation between the low and high bandgap PV cells at the receiver plane using the wavelength that corresponds to the bandgap for the high bandgap cell. This wavelength will be referred to as the transition wavelength $\lambda_{T}$. As

can be seen from the grating equation, wavelengths shorter than $\lambda_{T}$ are diffracted at smaller angles with respect to the hologram normal, while longer wavelengths within the spectral band are diffracted at larger angles with respect to the normal. The width of the VHL $\left(\mathrm{W}_{\mathrm{h}}\right)$ and the separation height $(\mathrm{h})$ are chosen to satisfy the high efficiency condition $(\mathrm{Q}>10)$ for the shortest wavelength in the diffracted spectral band. The width and shape of the diffracted spectral band are controlled by varying the exposure and processing conditions of the volume holographic recording material to chirp and shear the grating refractive index modulation function. The material selected for this process is dichromated gelatin which provides a great deal of flexibility for modifying the refractive index profile ${ }^{9,10}$.

A volume holographic lens was fabricated in a dichromated gelatin (DCG) film using the experimental setup shown in Fig. 5. A cylindrical lens is used in one arm of the interferometer to produce a focused beam approximately 1.0 
$\mathrm{cm}$ from the center of the hologram. The hologram is designed for a transition wavelength of $663 \mathrm{~nm}$ and recorded with light from a frequency doubled Nd:YAG laser $(532 \mathrm{~nm})$. The wavelength difference between the recording and reconstruction wavelengths are compensated by rotating the hologram normal by $7^{\circ}$ with respect to a normally incident beam which also includes the effect of swelling of the emulsion during the DCG film processing. The preexposure thickness of the DCG film is $16.0 \mu \mathrm{m}$ and $20.0 \mu \mathrm{m}$ after processing. The hologram exposure energy density is $750 \mathrm{~mJ} / \mathrm{cm}^{2}$.

After processing the diffraction efficiency of the VHL was measured at different points across the hologram aperture with three different laser wavelengths $(457 \mathrm{~nm}, 532 \mathrm{~nm}$, and $632.8 \mathrm{~nm}$ ). The resulting diffraction efficiencies are shown in Fig. 6. The results show high diffraction efficiency ( $>70 \%)$ across most of the hologram aperture over a significant spectral range using this simple construction method.

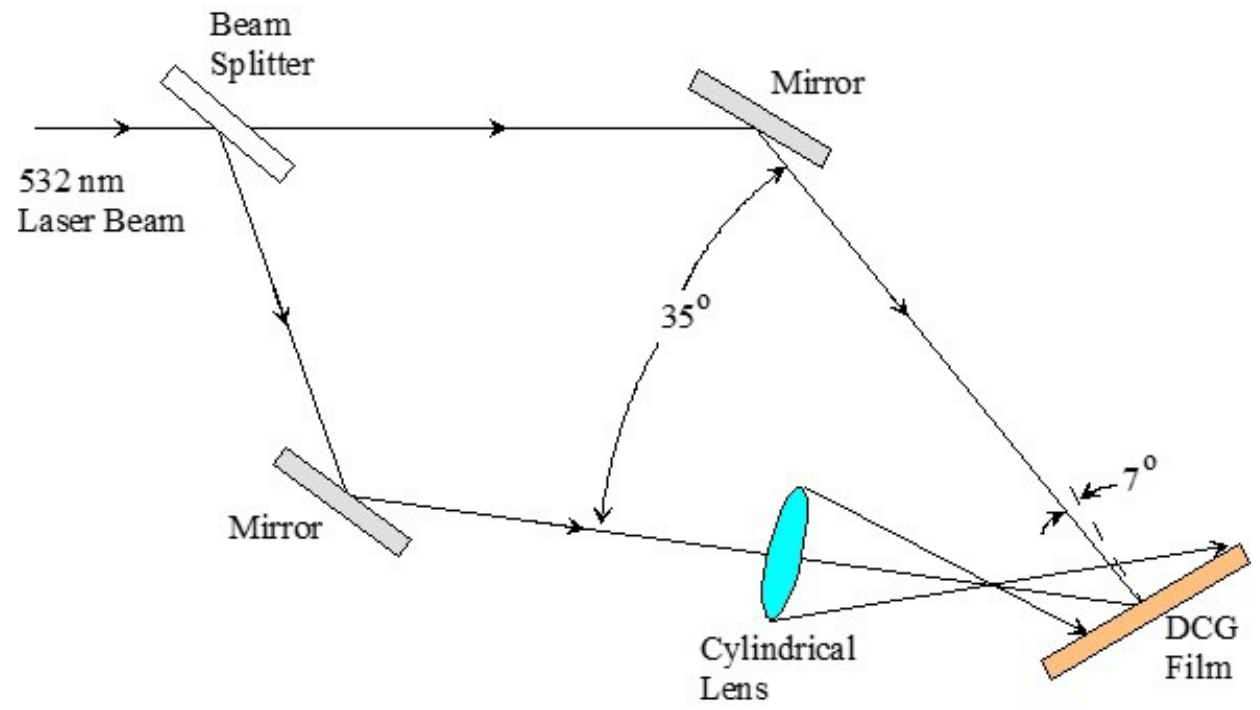

Fig. 5. Geometry for recording the VHL in dichromated gelatin film for use in a spectrum splitting module.

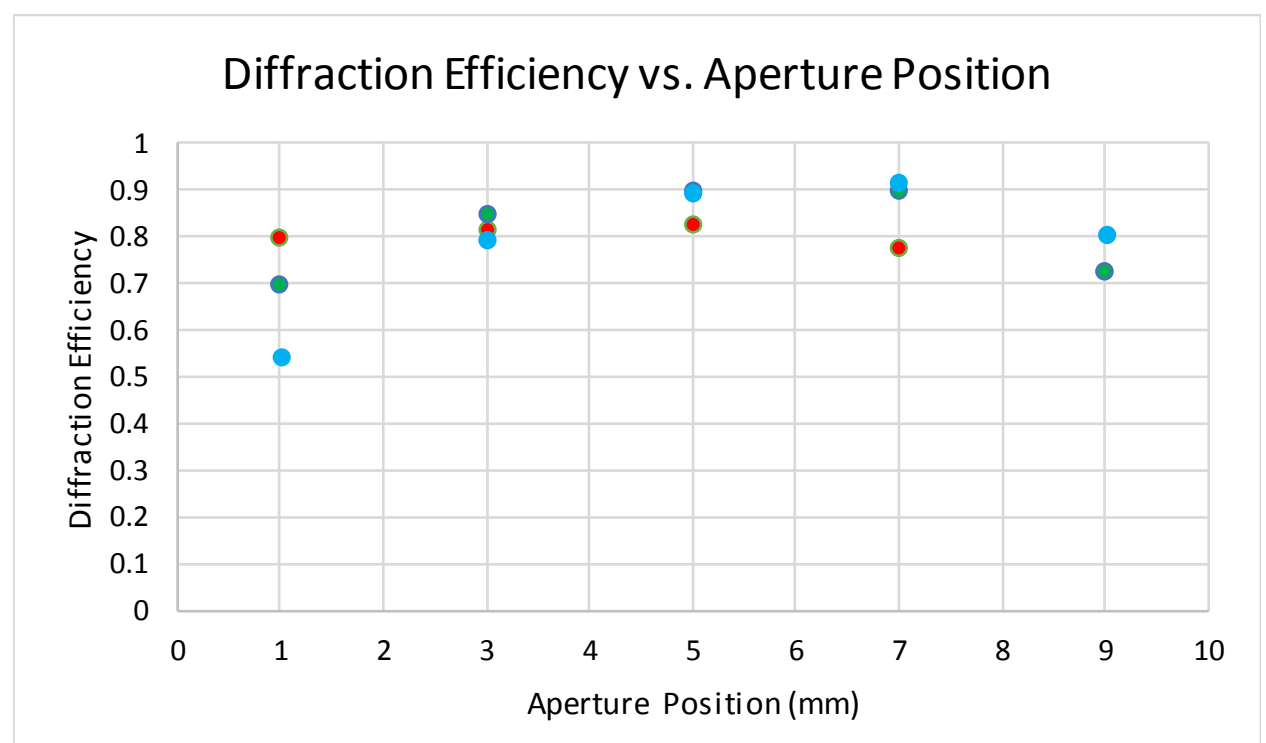

Fig. 6. Diffraction efficiency measured across the aperture of the volume holographic lens at $457 \mathrm{~nm}$ (blue), $532 \mathrm{~nm}$ (green) and $632.8 \mathrm{~nm}$ (red) laser wavelengths. 


\section{VHL MODULE CONFIGURATION}

The parameters shown in Table 1 were used in the design of a volume holographic lens module. The system height and width were chosen to be consistent with standard single PV cell modules. The hologram parameters were selected to satisfy high diffraction efficiency (i.e. Q > 10 across the lens aperture). The PV cells were chosen to maximize IoBB and the cell dimensions and concentration ratios to complement the hologram efficiency and module size requirements.

\begin{tabular}{|c|c|}
\hline \multicolumn{2}{|c|}{ Table 1: Module Properties } \\
\hline System Height & $1.00 \mathrm{~cm}$ \\
\hline Unit Cell Width & $1.29 \mathrm{~cm}$ \\
\hline Volume Holographic Lens Parameters \\
\hline Transition Wavelength & $663 \mathrm{~nm}$ \\
\hline \multicolumn{2}{|c|}{ PV Cell Properties } \\
\hline Hologram Film Thickness (After Processing) & $20 \mu \mathrm{m}$ \\
\hline Refractive Index Modulation & $0.077<\Delta \mathrm{n}<0.084$ \\
\hline Low Bandgap PV Cell (Silicon) & $24.4 \%$ \\
\hline High Bandgap PV Cell (InGaP) & $20.8 \%$ \\
\hline InGaP Cell width & $5.3 \mathrm{~mm}$ \\
\hline Silicon Cell width & $7.6 \mathrm{~mm}$ \\
\hline Geometrical CR (InGaP) & $2.43 \mathrm{X}$ \\
\hline Geometrical CR (Silicon) & $1.70 \mathrm{X}$ \\
\hline
\end{tabular}

A combination of raytracing and rigorous coupled wave analysis was used to determine the spectral optical efficiency of normally incident light. In addition, the spectral optical efficiency of diffuse illumination was modeled by evaluating rays that are incident over a range of $180^{\circ}$. Since the hologram is Bragg selective, rays incident at angles far from the Bragg condition are not diffracted by the hologram and illuminate the PV cell surfaces without separating spectral components.

Simulations were performed for AM1.5 spectral irradiance conditions and optimized VHL parameters to determine the direct normal incidence (DNI) conversion efficiency, IoBB, and the energy yield for a geographical location with high DNI (Tucson, AZ) and high diffuse illumination (Seattle, WA). The best performing single bandgap cell for this system is the silicon cell (24.4\%) since its spectral response has greater overlap with the AM 1.5 spectrum. The results are summarized in Table 2 and show that the power conversion IoBB for the VHL spectrum splitting system is $24 \%$, the IoBB energy yield for the high DNI condition is nearly $20 \%$, and $18 \%$ for the low DNI location.

\begin{tabular}{|c|c|}
\hline \multicolumn{2}{|c|}{ Table 2: AM 1.5 Irradiance/ 1000W/m² } \\
\hline Spectrum Splitting: Conversion Efficiency & $30.2 \%$ \\
\hline Improvement over Best Bandgap (Ideal Filter) & $\mathbf{2 4 . 0 \%}$ \\
\hline Improvement over Best Bandgap (Experimental VHL) & $\mathbf{1 5 . 0 \%}$ \\
\hline \multicolumn{2}{|c|}{ Annual Energy Yield in Tucson, AZ } \\
\hline Total Incident Insolation (2-axis tracking) & $3068.25 \mathrm{~kW}-\mathrm{hr}$ \\
\hline Spectrum Splitting: Converted Insolation & $1057.34 \mathrm{~kW}-\mathrm{hr}$ \\
\hline
\end{tabular}




\begin{tabular}{|c|c|}
\hline Spectrum Splitting: Conversion Efficiency & $34.5 \%$ \\
\hline Improvement over Best Bandgap (Ideal Filter) & $\mathbf{1 9 . 9 \%}$ \\
\hline \multicolumn{2}{|c|}{ Improvement over Best Bandgap (Experimental VHL) } \\
\hline \multicolumn{2}{|c|}{ Annual Energy Yield in Seattle, WA } \\
\hline Total Incident Insolation (2-axis tracking) & $1736.73 \mathrm{~kW}-\mathrm{hr}$ \\
\hline Spectrum Splitting: Converted Insolation & $565.18 \mathrm{~kW}-\mathrm{hr}$ \\
\hline Spectrum Splitting: Conversion Efficiency & $32.5 \%$ \\
\hline Improvement over Best Bandgap (Ideal Filter) & $\mathbf{1 8 . 2} \%$ \\
\hline Improvement over Best Bandgap (Experimental VHL) & $\mathbf{1 0 . 5 \%}$ \\
\hline
\end{tabular}

The performance of the experimental VHL was evaluated by using the measured spectral and spatial characteristics as the filter function for the system. In this case the power conversion IoBB is $15 \%$, the energy yield IoBB approximately $13 \%$ for the high DNI location, and $10.5 \%$ for the low DNI location. Further improvements in the experimental VHL performance can be realized by segmenting the aperture and control the exposure parameters to optimize the index modulation across the aperture.

\section{ACKNOWLEDGEMENTS}

The authors would like to thank the National Science Foundation (NSF) and Department of Energy (DOE) (EEC1041895, ECCS-1405619) for support of this work.

\section{REFERENCES}

[1] Shockley, W., and Queisser, H. J., "Detailed balance limit of efficiency of p-n junction solar cells," Journal of Applied Physics, 32(3), 510-519 (1961).

[2] Polman, A., and Atwater, H. A., "Photonic design principles for ultrahigh-efficiency photovoltaics," Nat. Mater. 11(3), 174-177 (2012).

[3] Gordon, M., Zhang, D., Vorndran, S., Russo, J. M., Luscombe, C. K., Shaheen, S. E., and Kostuk, R. K., "Planar holographic spectrum-splitting PV module design," High and Low Concentrator Systems for Solar Electric

Applications VII 8468(520), K. VanSant and A. P. Plesniak, Eds., 846808-9 (2012).

[4] Russo, J. M., et al., "Spectrum splitting metrics and effect of filter characteristics on photovoltaic system performance," Opt. Express, 22(102), A528-A541 (2014).

[5] Russo, J. M., Zhang, D., Gordon, M., Vorndran, S. D., Wu, Y., and Kostuk, R. K., "Grating-over-lens concentrating photovoltaic spectrum splitting systems with volume holographic optical elements," Proc. SPIE 8821, 882106-1:8 (2013).

[6] Zhang, D., Gordon, M., Russo, J. M., Vorndran, S., and Kostuk, R. K., "Spectrum-splitting photovoltaic system using transmission holographic lenses," J. of Photonics for Energy, 3(1), 034597-1:12 (2013).

[7] Angel, R. P., et al., "Tandem photovoltaic module with diffractive spectral separation," June 122015. Provisional Patent Application. Serial No. 62/1775,051.

[8] Kogelnik, H.. "Coupled wave theory for thick hologram gratings," Bell Syst. Tech. J., 48(9), 2909-2947 (1969).

[9] Stojanoff, C. G., et al., "Conceptual design and practical implementation of dichromated gelatin films as an optimal holographic recording material for large-format holograms," Proc. SPIE 2042, 301-311 (1994).

[10] Stojanoff, C. G., et al., "Design, fabrication, and integration of holographic dispersive solar concentrator for terrestrial applications," Proc. SPIE 1536, 206-214 (1991). 